\title{
Beam-beam effects of gear changing in ring-ring colliders
}

\author{
Yue Hao, ${ }^{*}$ V. N. Litvinenko, and V. Ptitsyn \\ Brookhaven National Laboratory, Upton, New York 11973, USA and \\ Department of Physics, Stony Brook University, Stony Brook, New York 11794, USA
}

(Received 9 September 2013; published 4 April 2014)

\begin{abstract}
In ring-ring colliders, the collision frequency determines the bunch structures, e.g., the time between the bunches in both rings should be identical. Because of relatively low relativistic speed of the hadron beam in sub-TeV hadron-hadron- and electron-ions colliders, scanning the hadron beam's energy would require either a change in the circumference of one of the rings, or a switching of the bunch (harmonic) number in a ring. The later would cause so-called gear changing, i.e., the change of the colliding bunches turn by turn. In this article, we study the difficulties in beam dynamics in this gear-changing scheme.
\end{abstract}

DOI: 10.1103/PhysRevSTAB.17.041001

PACS numbers: 29.27.-a

\section{INTRODUCTION}

In sub-TeV hadron colliders [1] and the ring-ring scheme for electron-ion colliders [2], scanning the energy of one of the hadron beams is desired. This need brings the challenge of synchronizing the two colliding beams. Since the hadron beams are not ultrarelativistic in the sub-TeV energy range, their velocity is significantly dependent upon their energy. To assure that both beams collide at a fixed interaction point (IP), the circumference of hadron ring must be adjusted when its energy changes. For instance, if one changes the hadron beam from $250 \mathrm{GeV}$ per nucleon $(\mathrm{GeV} / \mathrm{n})$ to $25 \mathrm{GeV} / \mathrm{n}$, its velocity changes about $0.07 \%$. Synchronizing the collision requires a proportional change of the ring's circumference. For a $3 \mathrm{~km}$ circumference, such an energy change corresponds to a change in pass length of about $2.1 \mathrm{~m}$. Altering the circumference would necessitate mechanically moving a large part (an arc) of the ring, so representing a major cost and operational obstacle.

To reduce or avoid such an adjustment, one can choose to change the rf harmonic number, $h$, of the ring, i.e., to change the number of bunches in the ring. This could allow the hadron beam to scan a discrete set of energies without adjusting the circumference. For the same example of a nominal energy $250 \mathrm{GeV} / \mathrm{n}$, where the rf harmonic number is 2500 , one can adjust the hadron beam's energy to $35.1 \mathrm{GeV} / \mathrm{n}, 24.9 \mathrm{GeV} / \mathrm{n}$, and $20.4 \mathrm{GeV} / \mathrm{n}$ by changing the harmonic number of either ring by 1,2, and 3 units, respectively, without changing the ring's circumference. If energies between those discrete values are required, a smaller adjustment of the circumference still would be required. In this example, one has to adjust the

\footnotetext{
*yhao@bnl.gov

Published by the American Physical Society under the terms of the Creative Commons Attribution 3.0 License. Further distribution of this work must maintain attribution to the author $(s)$ and the published article's title, journal citation, and DOI.
}

circumference of the ring by $1.05 \mathrm{~m}$, for the entire energy range between $250 \mathrm{GeV} / \mathrm{n}$ and $35.1 \mathrm{GeV} / \mathrm{n}$. If the rings' circumferences are equal and the rf harmonic numbers are not, one rf bucket in one of them will overlap with multiple rf buckets in the other ring in successive collision. Therefore the bunch in one ring will collide with multiple bunches in the other ring in different turns of revolution.

This asymmetric collision pattern could introduce complications with the beam dynamics. In this paper, we concentrate on exploring the consequence of these asymmetric patterns in two rings, including a multibunch offset/ dipole instability (Sec. II) and a multibunch beam-size/ quadrupole instability (Sec. III) due to the gear-changing beam-beam collision pattern, as well as the single particle dynamics at the presence of an abort gap in the opposing bunch train (Sec. IV).

\section{MULTIBUNCH, DIPOLE MOMENT ANALYSIS}

One possible side effect of this asymmetric collision pattern is the instability of the centroid of both beams. This topic is first studied in [3]. In this section, we present an alternative matrix method to study this problem; it easily can be extended to the case of large bunch numbers in both rings.

We will consider two rings of collider that have different bunch numbers, $N_{1}$ and $N_{2}\left(N_{2}>N_{1}\right)$, respectively. They are evenly distributed in the corresponding rf buckets. No bunch gap is considered herein. The time separations between the bunches are the same in both rings, so that the bunches are synchronized to collide at the IP. In this case, one bunch will collide not only with a single bunch in the opposing ring, but with $N_{c}$ bunches successively, where $N_{c}=\operatorname{LCM}\left(N_{1}, N_{2}\right) / N_{1}$. LCM () denotes the least common multiple of the arguments. If $N_{1}$ and $N_{2}$ are relatively prime numbers, the bunch will collide with all bunches of the opposing beam. We also assume that the optics functions at IP of the two rings are the same and the 
two colliding beams have the same emittance and intensity. Therefore the beam-beam parameters, $\xi$, for all bunches are the same.

The dipole moments of each bunch in ring 1 will affect the $N_{c}$ bunches in the other rings within the $N_{c}$ turns. The dipole moments must be stable in these collision processes. To characterize the dipole moment dynamics of $N_{1}+N_{2}$ bunches, we need to establish an equivalent "one-turn" matrix of a repetitive pattern for our analysis. The matrix is a $2\left(N_{1}+N_{2}\right)$ square matrix to denote the centroid motion of all the bunches. Accordingly, we have to calculate the matrix for successive $\operatorname{LCM}\left(N_{1}, N_{2}\right)$ collisions that corresponds to $\operatorname{LCM}(N 1, N 2) / N_{1}$ turns for beam 1 ; meanwhile, beam 2 finishes $\operatorname{LCM}(N 1, N 2) / N_{2}$ turns. When $N_{1}$ and $N_{2}$ are large and relative primes, it is very time consuming to construct this matrix for the repetitive pattern.

However, we found that we could simplify the calculation via the cyclic permutation matrix $P_{T}$ [Eq. (5)] that enables us readily to establish such a one-turn matrix. The effective $2\left(N_{1}+N_{2}\right) \times 2\left(N_{1}+N_{2}\right)$ one-turn total matrix $M_{T}$ has the form

$$
M_{T}=P_{T} M_{k} M_{\beta},
$$

in which, the betatron oscillation matrix $M_{\beta}$ is constructed as follows:

$$
M_{\beta}=\left(\begin{array}{ccc}
I\left(N_{1}\right) \otimes M\left(\theta_{1}\right) & 0 & 0 \\
0 & I\left(N_{1}\right) \otimes M\left(\theta_{2}\right) & 0 \\
0 & 0 & I\left(2 N_{2}-2 N_{1}\right)
\end{array}\right) .
$$

Here, $M(\theta)$ is the $2 \times 2$ one-turn matrix at the IP of both rings with $\theta_{1}$ and $\theta_{2}$, respectively, as the one-turn phase advance of two rings, respectively, symbol $\otimes$ denotes the matrix outer product.

$M_{k}$ is the linearized matrix for the beam-beam kick of the $i$ th bunch $\left(i=1 \ldots N_{1}\right)$ of beam 1 with the $i$ th one of beam 2. $M_{k}$ can be established by changing the following elements from a unit matrix,

$$
\begin{aligned}
M_{k}[2 i, 2 i-1] & =1 / f_{b b}, \\
M_{k}\left[2\left(N_{1}+i\right), 2\left(N_{1}+i\right)-1\right] & =1 / f_{b b}, \\
M_{k}\left[2 i, 2\left(N_{1}+i\right)-1\right] & =-1 / f_{b b}, \\
M_{k}\left[2\left(N_{1}+i\right), 2 i-1\right] & =-1 / f_{b b},
\end{aligned}
$$

with the focal length of the beam-beam interaction defined as $1 / f_{b b}= \pm 4 \pi \xi / \beta$, where the positive sign corresponds to the same sign of charge of the two colliding particles. The structure of matrix $M_{\beta}$ and $M_{k}$ shows that the betatron oscillation and the beam-beam kick apply to all the bunches in beam 1, but only the first $N_{1}$ bunches of beam 2 .
The rotation matrix $P_{T}$ is constructed as follows:

$$
P_{T}=\left(\begin{array}{cc}
I\left(2 N_{1}\right) & 0 \\
0 & P^{N_{2}-N_{1}}\left(N_{2}\right) \otimes I(2)
\end{array}\right),
$$

where $I(n)$ and $P(n)$, respectively, are the identity matrix and the cyclic permutation matrix of rank $n$. The permutation matrix $P$ reads

$$
P=\left(\begin{array}{ccccc}
0 & 0 & \ldots & 0 & 1 \\
1 & 0 & \ldots & 0 & 0 \\
0 & \ddots & \ddots & \vdots & \vdots \\
\vdots & \ddots & \ddots & 0 & 0 \\
0 & \ldots & 0 & 1 & 0
\end{array}\right),
$$

where the eigenvalues of $P$ with rank $k$ are $e^{i 2 m \pi / k}$, $m=0,1, \ldots, k-1$. The rotation matrix $P_{T}$ will rotate the beam 2 so that the remaining $N_{2}-N_{1}$ bunches will interact, in turn, with the opposing bunches in beam 1 . This method avoids our having to construct a complicated matrix for the $\operatorname{LCM}\left(N_{1}, N_{2}\right)$ collisions without any approximation, as seen in the Appendix.

We first discuss the case without collision, i.e., the matrix $M_{k}$ is an identity matrix. The eigenvalues of the matrix $M_{T}$ have $N_{1}$ degenerate pairs of $e^{ \pm i \theta_{1}}$ that come from the tune of ring $1\left(\theta_{1}=2 \pi \nu_{1}\right)$. However the eigenvalues contributed from ring 2 has unusual form that reads $e^{ \pm i \theta_{2 j}}, \theta_{2 j}=$ $2 \pi\left(N_{1} \nu_{2}+j\right) / N_{2}, \quad j=\operatorname{GCD}\left(N_{1}, N_{2}\right), \ldots, N_{2}$, where $\operatorname{GCD}\left(N_{1}, N_{2}\right)$ is the greatest common divider of two bunch numbers. As expected, the absolute values of all eigenvalues are one. Hereafter, we limit our discussion in the cases when $N_{1}$ and $N_{2}$ are relative prime numbers.

The effective tunes extracted from the total matrix $M_{T}$ are defined as $\Xi_{i}\left(i=1, \ldots, N_{1}+N_{2}\right)$. When the beambeam parameter is zero, there are $N_{1}$ degenerate tunes $\Xi_{1, i}=\nu_{1}\left(i=1, \ldots, N_{1}\right)$ from the tune of ring 1 , and $N_{2}$ distinct tunes $\Xi_{2, j}=\left(N_{1} \nu_{2}+j\right) / N_{2}\left(i=1, \ldots, N_{2}\right)$ from ring 2. If the beam-beam interaction is included, $\Xi_{1}$ and the $\Xi_{2 j}$ will shift according to the beam-beam parameter. The system becomes unstable when integer or half integer resonances happen and one or more eigenvalues of the matrix $M_{T}$ have absolute value larger than one. However, the resonance condition in this asymmetric collision case is more complicated. We also note that, in this asymmetric scheme, there exist $\sigma$ modes similar to the symmetric collision case. The $\sigma$ modes occurs when $\Xi_{1}(\xi=0)=\Xi_{2, j}(\xi=0)$. The tune of this mode remains intact with beam-beam interaction of any strength.

Figure 1 illustrates the instability of the total map $M_{T}$, occurring when two eigentunes merge. In this example, the attractive beam-beam force is used which corresponds to the case of the electron-ion colliders. We choose the number of bunches 3 and 4, respectively, in the two rings 

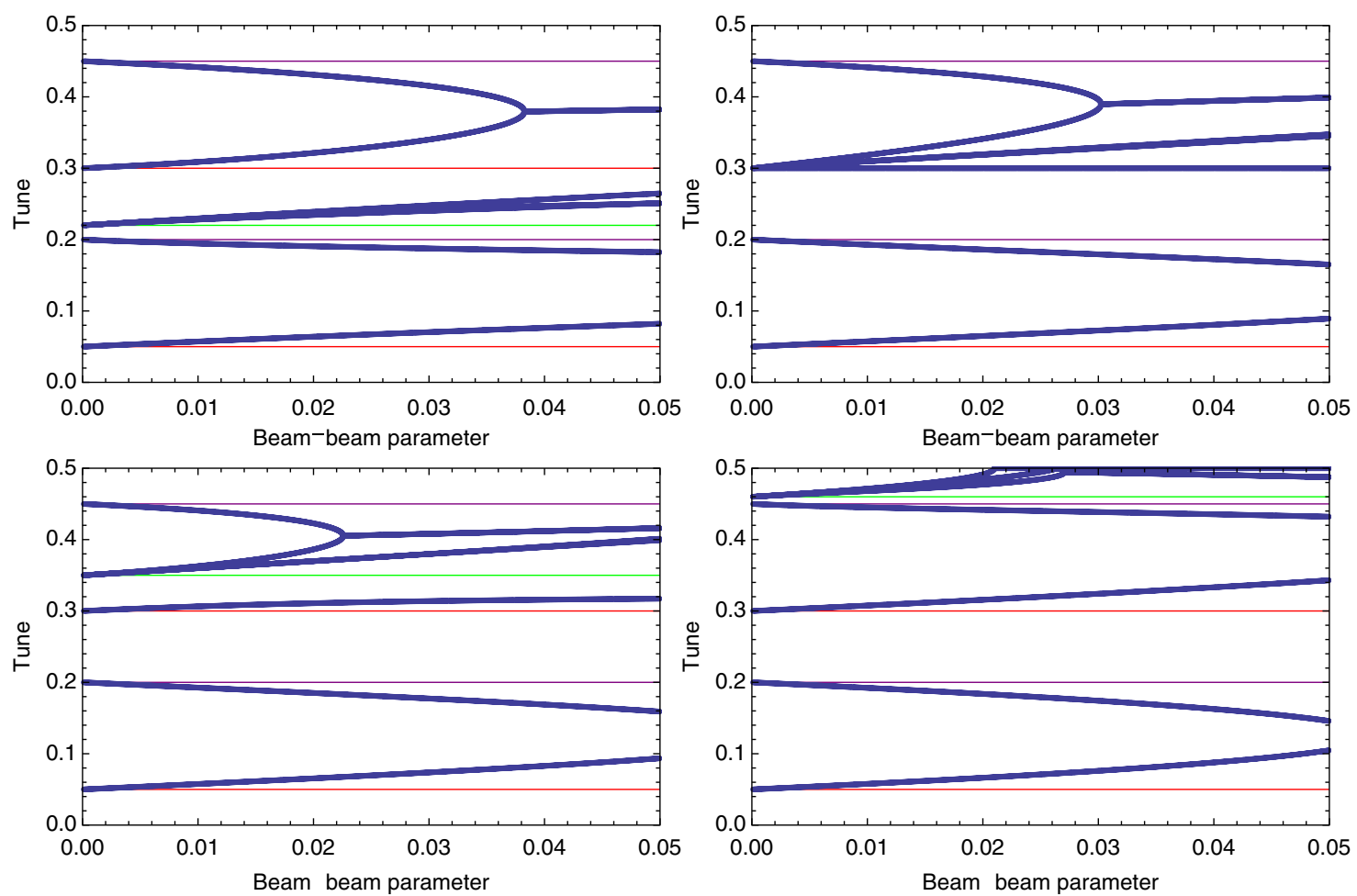

FIG. 1. The tunes extracted from matrix $M_{T}$ with different beam-beam parameters $\xi$. The vertical grid lines represent the tunes $\Xi_{i}$ when there is no beam-beam interaction. The green one denotes $\Xi_{1}$ and the red and purple ones represent the $\Xi_{2}$ or $1-\Xi_{2}$ in the range $[0,0.5]$, respectively. All figures have the number of bunches in two ring as $\left(N_{1}, N_{2}\right)=(3,4)$ with different tunes. The tunes are the following: top left $\left(\nu_{1}, \nu_{2}\right)=(0.22,0.4)$; top right $\left(\nu_{1}, \nu_{2}\right)=(0.3,0.4)$; bottom left $\left(\nu_{1}, \nu_{2}\right)=(0.35,0.4)$ and bottom right $\left(\nu_{1}, \nu_{2}\right)=(0.46,0.4)$.

and fixed the tune of the second ring as $\nu_{2}=0.4$. Therefore the effective tune $\Xi_{2}=\{0.3,0.55,0.8,0.05\}$ when the beam-beam parameter is zero, and the tune of ring $1 \Xi_{1}=$ $\nu_{1}$ is varied to explore the structure of the tune map. In the top left figure, where $\nu_{1}=0.22$, the instability happens when the two tunes of $\Xi_{2,1}=0.3$ and $\Xi_{2,2}=0.55$ merges at beam-beam parameter $\xi \sim 0.036$, which is referred to as the sum resonance $\Xi_{2,1}+\Xi_{2,2} \sim 1$, i.e., the half-integer resonance of $N_{1} \nu_{2}$. The top right figure illustrates the appearance of the $\sigma$ mode when $\nu_{1}=0.3$ was chosen. We can observe that this tune remains intact when the beambeam parameter increases. The bottom left figure reflects the sum resonance of $\Xi_{1}+\Xi_{2,2} \sim 1$, which corresponds to the integer number resonance of $N_{1} \nu_{2}+N_{2} \nu_{1}$, when $\nu_{1}$ is set to 0.35 . A trivial case of half integer resonance at $\Xi_{1} \sim$ 0.5 is shown in the bottom right one with $\nu_{1}=0.46$.

These resonances are based on the integer turns of ring 1 . Therefore only the half integer resonance of $N_{1} \nu_{2}$ and $\nu_{1}$ are observed directly. If we rewrite the total matrix based on the integer turns of ring 2, the other two symmetric counterparts $N_{2} \nu_{1}$ and $\nu_{2}$ can be found.

From the above discussion, we learned that, in this asymmetric collision pattern, there are many more linear resonances to be avoided than in a symmetric pattern for any selected working point in the tune diagram. The sum resonances may occur between the $\Xi_{1}$ and $1-\Xi_{2, j}$ or $\Xi_{2, i}$ and $1-\Xi_{2, j}$. The spacing between $\Xi_{2}$ is $1 / N_{2}$. If $N_{2}$ is a large number, it will strongly limit the choice of the tunes in both rings and the maximum beam-beam parameter, so to avoid linear resonances. In the linear beam-beam tune shift approximation, $d \Xi / d \xi=1$; therefore we have to limit the beam-beam tune shift to $\xi \lesssim 1 / 2 N_{2}$, as well as to select proper tunes to avoid the sum resonance. The working point in storage rings is determined by many other factors, such as the nonlinear resonances, dynamic aperture optimization, as well as spin resonances for polarized beam colliders. Our study indicates that a fine-tuning of the working points is also required to avoid a dense net of resonance lines separated by approximately $1 / N_{2}$. In Fig. 2 , we vary $N_{1}$ and beam-beam parameter $\xi$ in a large range and optimize both tunes of the rings, $\nu_{1}$ and $\nu_{2}$, around 0.2 to minimize the largest modulus of the eigenvalue of the matrix $M_{T}$ for an individual $N_{1}$. In these processes, we fixed $N_{2}=N_{1}+1$. The color map and the contour lines in Fig. 2 indicates optimized largest modulus values, and therefore the stable region is defined by the contour line of modulus equaling 1 . As expected the boundary of the stable region is a linear line in the logarithmic scale, which indicates $\xi_{\max } \sim 1 / N_{1} \sim 1 / N_{2}$. The slope of this line is about 0.25 , as derived by linear regression; therefore, the 


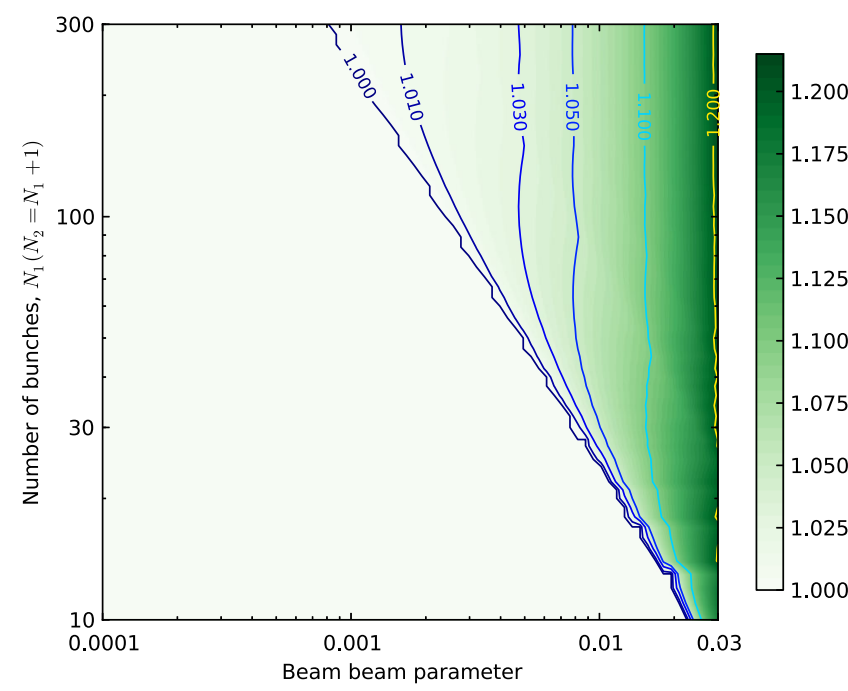

FIG. 2. The largest module of the eigenvalues of $M_{T}$ for different beam-beam parameters and number of bunch in ring one $N_{1}$, while $N_{2}=N_{1}+1$. Both tunes in two rings are optimized around 0.2 for each $N_{1}$.

stability boundary reads $\xi_{\max } \approx 1 / 4 N_{1}$. For bunch numbers of $N_{1}=100$ and $N_{2}=101$, the maximum beam-beam parameter is only $2.5 \times 10^{-3}$, which is much smaller than the beam-beam parameter realized in both hadron and lepton colliders in a symmetric collision pattern. It is worthwhile to note that, if both bunches have Gaussian transverse distribution, the beam-beam focal strength is half of the value of a flat-top one, which is assumed in Eq. (3). Therefore, the maximum beam-beam parameter discussed above is underestimated by a factor of 2 if both beams have Gaussian transverse distribution instead of the flat-top one $[3,4]$.

To restore the beam-beam parameters of the asymmetric collision pattern to the symmetric one, a bunch-by-bunch transverse feedback system (transverse damper) is required. The largest modulus plotted in Fig. 2 helps one determine the damping time of such damper. To restore the limit of the typical beam-beam parameter in a hadron collider $(\xi \sim 0.02)$, the damping time of such damper must be less than 10 turns for a large $N_{1}(\geq 100)$, since the largest modulus is around 1.1 for those bunch numbers. The radiation damping of the electron beam is not helpful, since the damping time is much longer than the requirement. The damping time of $\sim 10$ turns is a very challenging requirement and, if it is possible, would require an ultralownoise pickup and kicker system in either the hadron or the electron rings.

\section{MULTIBUNCH, QUADRUPOLE MOMENT ANALYSIS}

In this section, we detail our findings from extending our study to the transverse beam size instability in this gear-changing collision pattern. Herein, we assume that the dipole offset of all bunches are damped to zero.

We are interested in the vector of second-order moment $V=\left(\left\langle x^{2}\right\rangle,-\langle x x \prime\rangle,\left\langle x^{2}\right\rangle\right)^{T}$ and its propagation under a linearized beam-beam force. In this case, the emittance of the beam is constant. Therefore, for a $2 \times 2$ betatron transfer map $M$, the transport matrix $R$ for the vector $V$ is well known,

$$
R(M)=\left(\begin{array}{ccc}
M_{11}^{2} & -2 M_{11} M_{12} & M_{12}^{2} \\
-M_{11} M_{21} & M_{11} M_{22}+M_{12} M_{21} & -M_{12} M_{22} \\
M_{21}^{2} & -2 M_{21} M_{22} & M_{22}^{2}
\end{array}\right) .
$$

The one-turn transport matrix for a second moment including linearized beam-beam effect is

$$
R(K) \times R\left(M_{0}\right),
$$

where

$$
M_{o}=\left(\begin{array}{cc}
\cos \mu_{\beta} & \beta^{*} \sin \mu_{\beta} \\
-\sin \mu_{\beta} / \beta^{*} & \cos \mu_{\beta}
\end{array}\right) \text { and } K=\left(\begin{array}{cc}
1 & 0 \\
1 / f_{b b} & 1
\end{array}\right) \text {. }
$$

Here $\mu_{\beta}$ is the transverse betatron tune and $f_{b b}$ is the focal length of the linearized beam-beam force. The matrix $R\left(M_{0}\right)$ has three eigenvalues as $\exp \left[ \pm 2 i \mu_{\beta}\right]$ and 1 . The matrix $R(K)$ has the explicit form,

$$
R(K)=\left(\begin{array}{ccc}
1 & 0 & 0 \\
-1 / f & 1 & 0 \\
1 / f^{2} & -2 / f & 1
\end{array}\right)
$$

We note that $f_{b b}$ is inversely proportional to the square of the rms beam size of the opposing beam provided that the beam is round. Therefore, we encounter the difficulty that the size of the two beams does not have a linear cross-talk as in the case of a centroid offset case, described in the previous section.

We may circumvent this problem by studying the evolution of infinitesimal second-order moment offset from the unperturbed vector. We may write $V=V_{0}+V$, where $V_{0}$ is an unperturbed vector $V_{0}=\left(\left\langle x^{2}\right\rangle_{0},-\langle x x\rangle_{0},\left\langle x \prime^{2}\right\rangle_{0}\right)^{T}$ and $V_{1}=\left(d\left\langle x^{2}\right\rangle,-d\langle x x \prime\rangle, d\left\langle x^{2}\right\rangle\right)^{T}$ is its first-order deviation. The unperturbed vector $V_{0}$ is related to the dynamic optics function as $V_{0}=(\beta, \alpha, \gamma)_{d} \varepsilon$, where $\varepsilon$ is the beam emittance. They are the ones that deviate from the design optics at IP due to the beam-beam force. If we assume the betatron phase advance is different in the two colliding rings, the dynamics optics functions also differ. Using $x$ and $y$ to denote two colliding bunches, we can write the transformation of the linearized beam-beam interaction as follows: 


$$
\begin{aligned}
d\left\langle x^{2}\right\rangle_{b b}= & d\left\langle x^{2}\right\rangle \\
-d\langle x x\rangle_{b b}= & -\frac{d\left\langle x^{2}\right\rangle}{f}+(-d\langle x x \prime\rangle)+\frac{\beta_{x}}{f \beta_{y}} d\left\langle y^{2}\right\rangle \\
d\left\langle\prime^{\prime 2}\right\rangle_{b b}= & \frac{d\left\langle x^{2}\right\rangle}{f^{2}}-2 \frac{-d\langle x x \prime\rangle}{f}+d\left\langle x^{2}\right\rangle \\
& -2 \frac{\alpha_{x}+\beta_{x} / f}{f \beta_{y}} d\left\langle y^{2}\right\rangle .
\end{aligned}
$$

Here the subscript $b b$ represents the deviation vector after the beam-beam interaction and the optics functions are the dynamics ones.

Similarly, we can build the one-turn map $M_{T}$ of the perturbed second-order moment as detailed in the previous section, $M_{T}=P_{T} M_{k} M_{\beta}$. We reuse all the symbols; however, it is straightforward that all matrices are $3\left(N_{1}+N_{2}\right)$ rank square matrices. $M_{\beta}$ is built from $R(M)$ by

$$
M_{\beta}=\left(\begin{array}{ccc}
I_{N_{1}} \otimes R_{M}\left[\theta_{1}\right] & 0 & 0 \\
0 & I_{N_{1}} \otimes R_{M}\left[\theta_{2}\right] & 0 \\
0 & 0 & I_{3 N_{2}-3 N_{1}}
\end{array}\right),
$$

where $I_{N}$ is the identity matrix with rank $N$, and $R_{M}[\theta]$ denotes $R\left[M_{0}(\theta)\right]$ for space saving.

The $M_{k}$ reads

$$
M_{k}=\left(\begin{array}{ccc}
I\left(N_{1}\right) \otimes R[K] & 0 & 0 \\
0 & I\left(N_{1}\right) \otimes R[K] & 0 \\
0 & 0 & I\left(3 N_{2}-3 N_{1}\right)
\end{array}\right)
$$

with additional terms modified as

$$
\begin{aligned}
M_{k}\left[3 i-1,3 i+3 N_{1}-2\right] & =\frac{\beta_{x}}{f \beta_{y}}, \\
M_{k}\left[3 i, 3 i+3 N_{1}-2\right] & =-2 \frac{\alpha_{x}+\beta_{x} / f}{f \beta_{y}}, \\
M_{k}\left[3 i+3 N_{1}-1,3 i+3 N_{1}-2\right] & =\frac{\beta_{y}}{f \beta_{x}}, \\
M_{k}\left[3 i+3 N_{1}, 3 i+3 N_{1}-2\right] & =-2 \frac{\alpha_{y}+\beta_{y} / f}{f \beta_{x}},
\end{aligned}
$$

with the index $i=1,2, \ldots, N_{1}$. We can construct the $P_{T}$ as follows:

$$
P_{T}=\left(\begin{array}{cc}
I\left(3 N_{1}\right) & 0 \\
0 & P^{N_{2}-N_{1}}\left(N_{2}\right) \otimes I(3)
\end{array}\right)
$$

where the permutation matrix $P$ has the same definition [Eq. (5)] as given in the last section.

The tunes of the second-order moment extracted from the matrix $M_{T}$ also have similar structures. Without beam-beam interactions, the tunes have the contribution from the ring 1 as $\Xi_{1, i}=2 \mu_{1}\left(i=1,2, \ldots, N_{1}\right)$ and the ones from the ring 2 as $\Xi_{2, j}=2 \mu_{2} N_{1} / N_{2}+j / N_{2}$, $\left(j=1,2, \ldots, N_{2}\right)$. There also are "dummy" tunes, which are $\Xi_{0}=j / N_{2},\left(j=1,2, \ldots, N_{2}\right)$. They are contributed from the eigenvalue 1 of the matrix $R\left(M_{0}\right)$ and remain constant at nonzero beam-beam interaction strength. When the beam-beam parameter is nonzero, the resonance can occur at certain beam-beam parameters, which can be predicted by the modulus and the arguments (tunes) of the eigenvalues of $M_{T}$. Figure 3 illustrates the resonance conditions of the sum resonances of $2 N_{2} \nu_{1}+2 N_{1} \nu_{2}$. We note that the stable region of the one-turn matrix $M_{T}$ may not be continuous as the beam-beam parameter increases, as shown in bottom graph of Fig. 3 .

The separation of $\Xi_{2}$ lines is $1 / N_{2}$, which is the same as the dipole analysis in the last section. The difference is that the beam-beam tune shift $d \Xi / d \xi$ is 2 instead of 1 , in the second-order moment case, when the beam-beam tune shift is small. Therefore, the maximum achievable beam-beam parameter is expected to be smaller than in the dipole case, before the first linear resonance is observed. However, there may be another stable region followed by an unstable region as shown in Fig. 3. Therefore, it is difficult to conclude a simple criterion of the stable system.
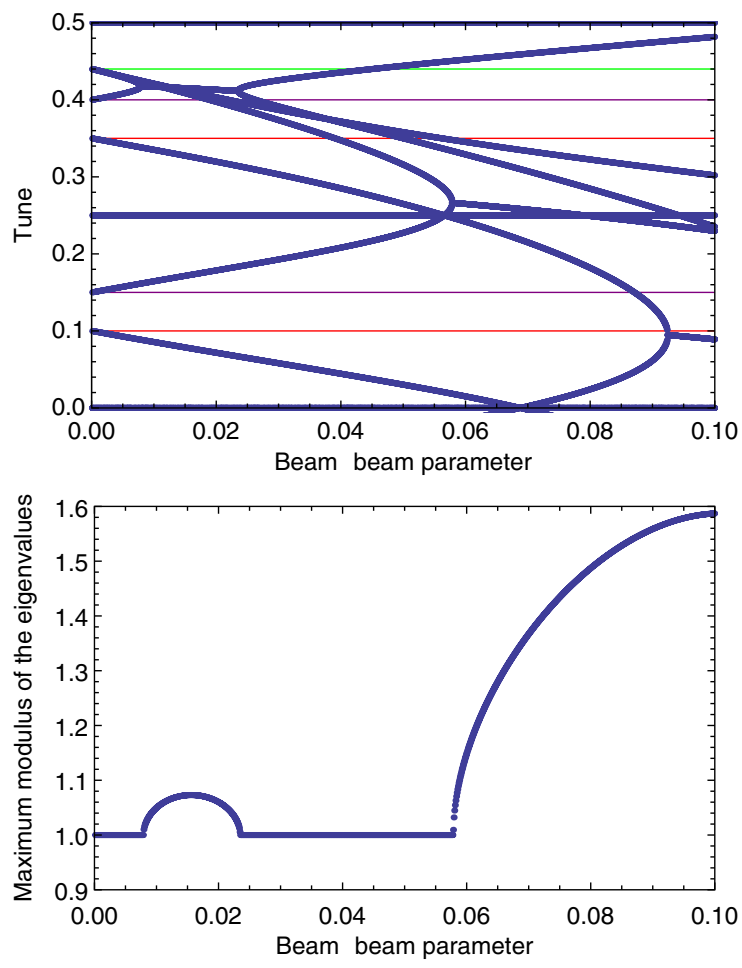

FIG. 3. The tunes extracted from second-order moment one-turn matrix $M_{T}$ (top) and the largest modulus of its eigenvalues (bottom) as a function of the beam-beam parameter. In this example, the number of bunches in two rings are $\left(N_{1}, N_{2}\right)=(3,4)$. The tunes are $\left(\nu_{1}, \nu_{2}\right)=(0.22,0.4)$. 


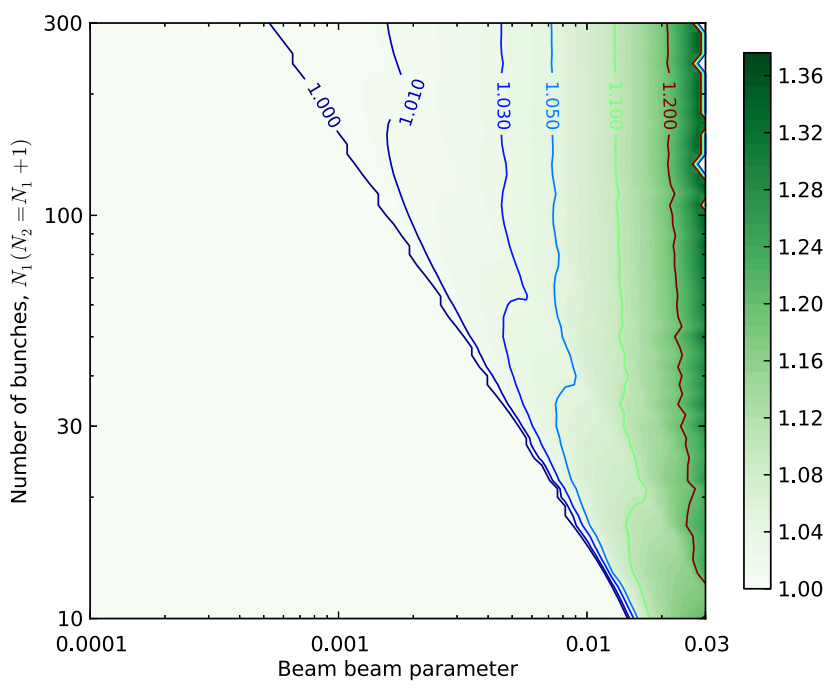

FIG. 4. The largest module of the eigenvalues of $M_{T}$ for different beam-beam parameters and number of bunches in ring one $N_{1}$, while $N_{2}=N_{1}+1$, for the second-order moment case. Both tunes in two rings are optimized around 0.2 for each $N_{1}$.

Figure 4 illustrates the color map of the optimized largest modulus of the one-turn matrix $M_{T}$. Both tunes are optimized in the vicinity of $\nu_{1,2} \sim 0.2$. The stable boundary also is a straight line in the logarithmic scale. We use the linear regression to obtain the stable boundary condition as $\xi_{\max } \approx 0.2 N_{1}$, which is, as expected, a tighter condition than the stable condition for the dipole offset case.

On this linearized model, we imposed two strong assumptions. First is that the model uses the perturbed vector of the second-order moment. Therefore even if oneturn $M_{T}$ is unstable, we only can conclude that the system will deviate from the unperturbed second-order moment of the beam, which, in a nonlinear system, does not necessarily lead to instability. Second, we assume the emittance of the beam is constant after the nonlinear beam-beam interaction and the beam will maintain a Gaussian distribution with different rms value. However, the emittance and the transverse distribution of the beam will change slowly under the nonlinear force especially when beam sizes of the two opposing beams do not match. Also in our linear model, we overestimated focusing/defocusing effect of the beam-beam force, since the particles with larger amplitude (beyond $2 \sigma$ in beam size) experience much smaller focusing/defocusing force than a linearized one used in the model. Therefore we are expecting the stabilization condition in a nonlinear simulation will be more relaxed than that predicted by this linear model. After scaled by a factor $(\leq 1)$, the maximum stable beam-beam parameter found in a nonlinear simulation is expected to be compared with that of the linear model.

We test the understanding of the accuracy of this model using a multibunch 1D (2D in phase space) simulation including nonlinear beam-beam force. In this model, the coordinates are denoted as $\left(r, r^{\prime}\right)$. The beam-beam interaction is represented by

$$
\Delta r^{\prime}=\frac{8 \pi \xi}{\beta^{*}} \frac{\sigma_{r, 0}^{2}}{r}\left[1-\exp \left(-\frac{-r^{2}}{2 \sigma_{r}^{2}}\right)\right]
$$

where $\xi$ is the design beam-beam parameter, $\sigma_{r, 0}$ is the initial beam size, which is the same for all bunches in both beams, and $\sigma_{r}$ is the rms beam size of the opposing beam before the beam-beam interaction of each turn. In this simulation, the initial beam size is set to $100 \mu \mathrm{m}$ and $\beta^{*}$ is $0.5 \mathrm{~m}$. The number of bunches in two rings are $\left(N_{1}, N_{2}\right)=(50,51)$. Each bunch has 1000 macroparticles to represent the colliding beam. The bunches are assumed to be thin disks, i.e., only transverse effects are considered. Also, chromatic effect is excluded from the simulation. In addition, we excluded the dipole effect by resetting the offset of the centroid of each bunch to ensure that the observation is focused on the changes in beam size of both beams. Figure 5 shows the results and compares them with the linear model. The top left figure shows the instability of multibunch beam size with the beam-beam parameter 0.015 . The top right one shows the luminosity reductions due to this instability at different $\xi$. We may observe losses as low as $\xi=0.003$, while the system has returned to a stable condition at $\xi=0.007$ and becomes unstable again at $\xi=0.01$. We then scanned the beam-beam parameter in the simulation and compared the finding with the linear matrix model. The model predicts the result very well except that we had to scale the beam-beam parameter in the simulation with a factor of 0.5 to achieve a better match. This disparity reflects our overestimation of the beam-beam focusing effect in the linear model.

Now, we fix the working point of both rings as 0.68 , and vary the number of bunches in ring $1\left(N_{1}\right)$ from 20 to 1000 , while keeping $N_{2}=N_{1}+1$. Figure 6 shows the luminosity reduction after 5000 turns as function of $N_{1}$. The luminosity loss tends to saturate at a very low level, $\sim 20$ times reduction at $\xi=0.01$ and $\sim 8$ times reduction at $\xi=0.005$, when the number of bunches is beyond 400 . The zigzag scattered points in this figure reflects that the fixed tune (0.68) is not optimized for different $N_{1}$. In this tune, the resonance strength is larger for some bunch number, while smaller for others $N_{1}$. The tune can be optimized by a range of $1 / N_{1}$ to minimized the unwanted resonances.

When the system becomes unstable, the luminosity drops quickly in the first few thousand turns, then stabilizes itself after that. The stabilization results from both the inverse quadratic relation of the beam-beam parameter as a function of the beam size and the nonlinearity of the beambeam field. In addition, we observe a slow luminosity loss (as shown in the bottom right of Fig. 5) after the selfstabilization. The loss is not observed in a symmetric scheme, $N_{1}: N_{2}=1: 1$, with the same beam-beam parameter. We use linear regression to obtain the speed of the luminosity loss as $2 \%$ every $100 \mathrm{~K}$ turns for this beambeam parameter. For a $3000 \mathrm{~m}$ circumference ring, the decline in luminosity will be $2 \%$ per second. For an 

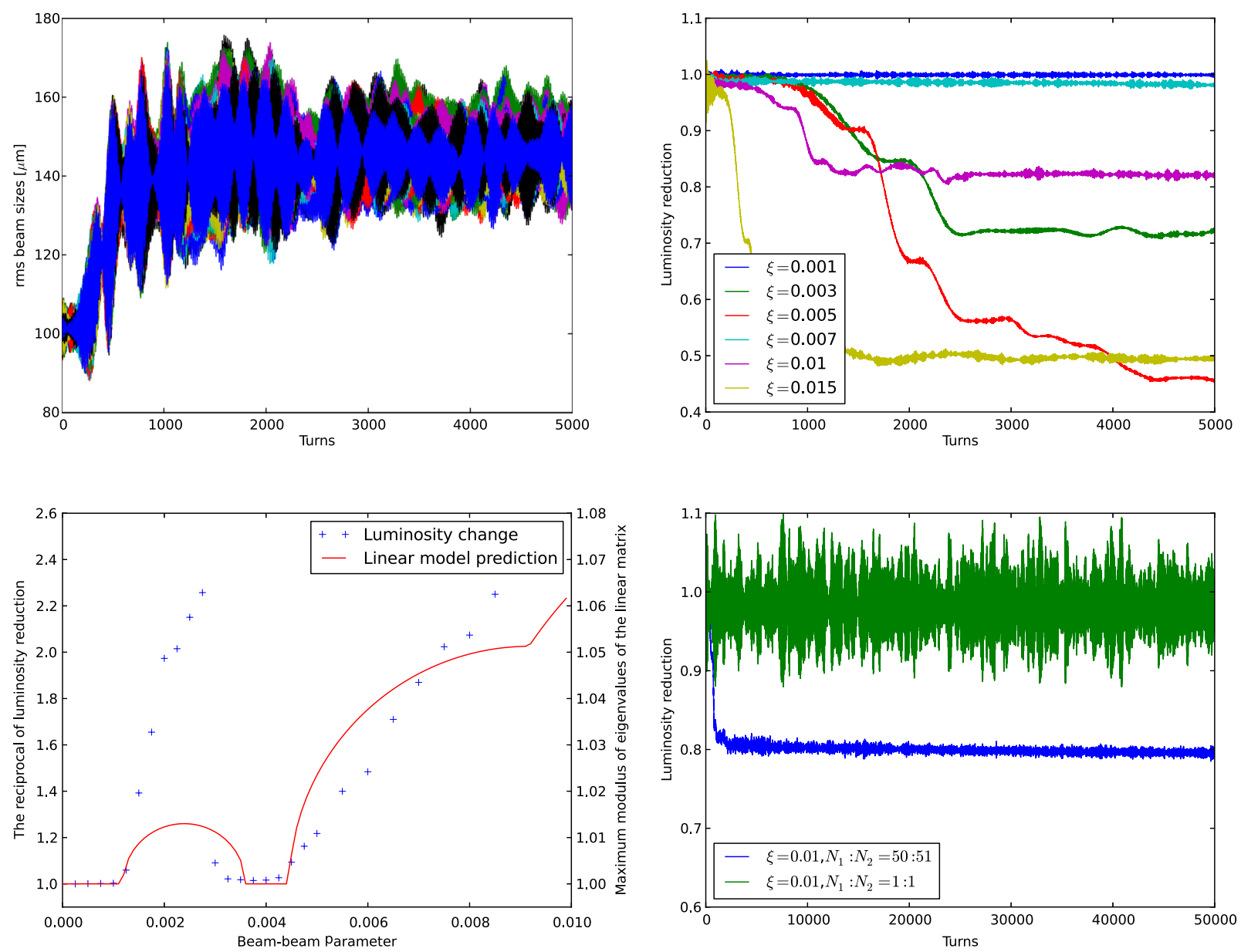

FIG. 5. The results of nonlinear $1 \mathrm{D}$ beam-beam simulation for $\left(N_{1}, N_{2}\right)=(50,51)$. Top left: the beam size evolutions for $\xi=0.015$, which indicates instability of the beam size. Top right: the luminosity reduction due to the second-order moment instability in asymmetric collision scheme. Bottom left: the reciprocal of luminosity reduction at the end of 5000 turns and the comparison with linear matrix model. The beam-beam parameter in the nonlinear simulation is scaled by 0.5 in this plot. Bottom right: comparison of the asymmetric and symmetric collision scheme. The tunes of two rings are all set to 0.68 to avoid lower order nonlinear resonances.

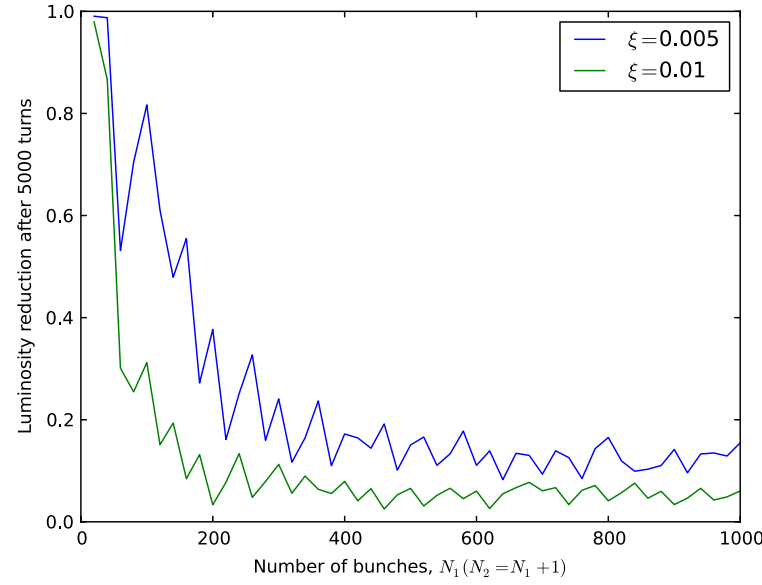

FIG. 6. Luminosity reduction as function of the bunch number $N_{1}$. electron-ion collider, the synchrotron radiation damping of the electron beam may suppress this slow loss. However, the hadron collider requires aggressive cooling technology to prevent this slow loss.

In summary, we used similar matrix method to predict instability of the second-order moment of the colliding bunches due to the asymmetric collision pattern. The 1D simulation confirms the prediction of the linear model that this instability prevents the choice of a reasonable beambeam parameter. This instability will entail a very rapid luminosity loss in milliseconds scale and cannot be overcome by a transverse damper as the dipole offset case in the previous section. Then the system is self-stabilized at a much lower luminosity than the design value and continues to suffer from a slow luminosity loss. It is worthwhile to note that for the same tune of ring 2, the effective tunes from $M_{T}$ are generally different in the dipole and quadrupole cases; therefore, it is impossible to find an optimized tune for both effects. 


\section{SINGLE BUNCH EFFECT WITH BUNCH GAP}

Finally, we studied the beam-beam effects in the presence of the bunch gap. In either ion or electron rings, some buckets have to be empty for various reasons such as the injection gap, eliminating ion trapping or electron cloud effects. We assume that the gap is in ring 2, which can hold $N_{2}$ bunches and that all gaps are positioned together, i.e., in the $N_{2}$ collisions, the bunch in ring 1 will meet $m$ opposing bunches and $n=N_{2}-m$ empty buckets. The linear matrix which includes the coherent beam-beam effect of the $N_{2}$ turns becomes

$$
M_{t}=M_{\beta}(n \phi)\left(M_{k}(\xi) M_{\beta}(\phi)\right)^{m},
$$

where $M_{\beta}$ is the transverse map with one-turn phase $\phi$ and $M_{k}$ is the matrix that represents the linearized beambeam kick.

The trace of $M_{t}$ determines the beam's stability with a linearized beam-beam interaction. Figure 7 plots the $\left|\operatorname{Tr}\left(M_{t}\right)\right| / 2-1$, which characterizes the unstable system via positive values. The result suggests that the matrix is an unstable one at many specific value of tunes with an approximate separation of $1 / N_{2}$. Those unstable tunes and their amplitude form an oscillating envelope. The valleys between peaks are the largest stable regions. The number of the envelope peaks equals the number of missing bunches, $n$.
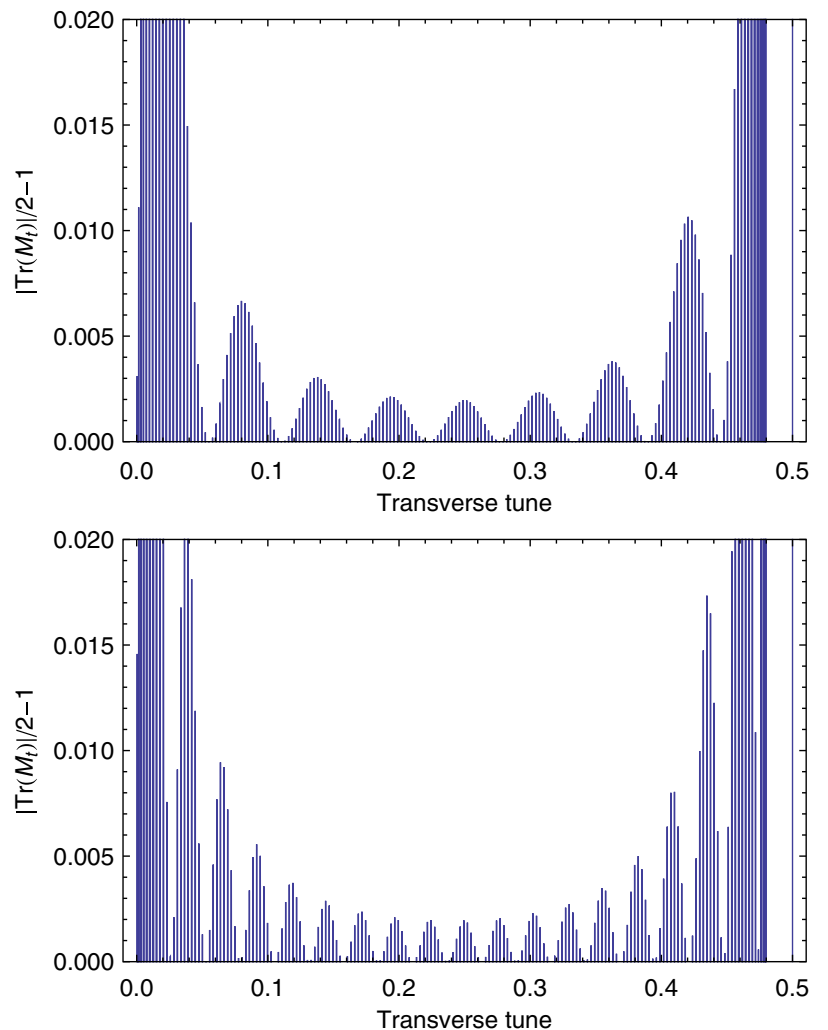

In detailing the linear beam dynamics, we always can find a proper working point that gives stable $N_{2}$ turn matrix $M_{t}$. However, the nonlinearity of the beam-beam force complicates our working point optimization, because the tune spread induced by the nonlinear force may cause the partial particles to fall in the unstable region. We used a 4D nonlinear beam-beam map to simulate the example depicted in Fig. 7. We also included chromaticity in both rings of unit 1 , assuming the rms energy spread was $3 \times 10^{-4}$. Figure 8 shows the emittance of such a bunch as a function of turns. The working points were chosen near the peaks or valleys as in our study of the linear model (bottom right of Fig. 7). With the on-peak working points (the cyan and the green curves), the growth rate of the beam emittance is the highest, approximately 5\% every 500 thousand turns. When the working points are chosen to be in the valleys (the stable region in linear study), the emittance growth is reduced dramatically. In this example, the emittance growth is not observed in the first 500,000 turns when the working points are close to 0.263 .

We conclude that in the asymmetric collision scheme with the presence of gaps in the bunch train, the single bunch has a very different stable condition from that in the symmetric case, where the bunch only collides with one opposing bunch. The choice of working points is limited to several small regions defined by the number of gap $\mathrm{rf}$
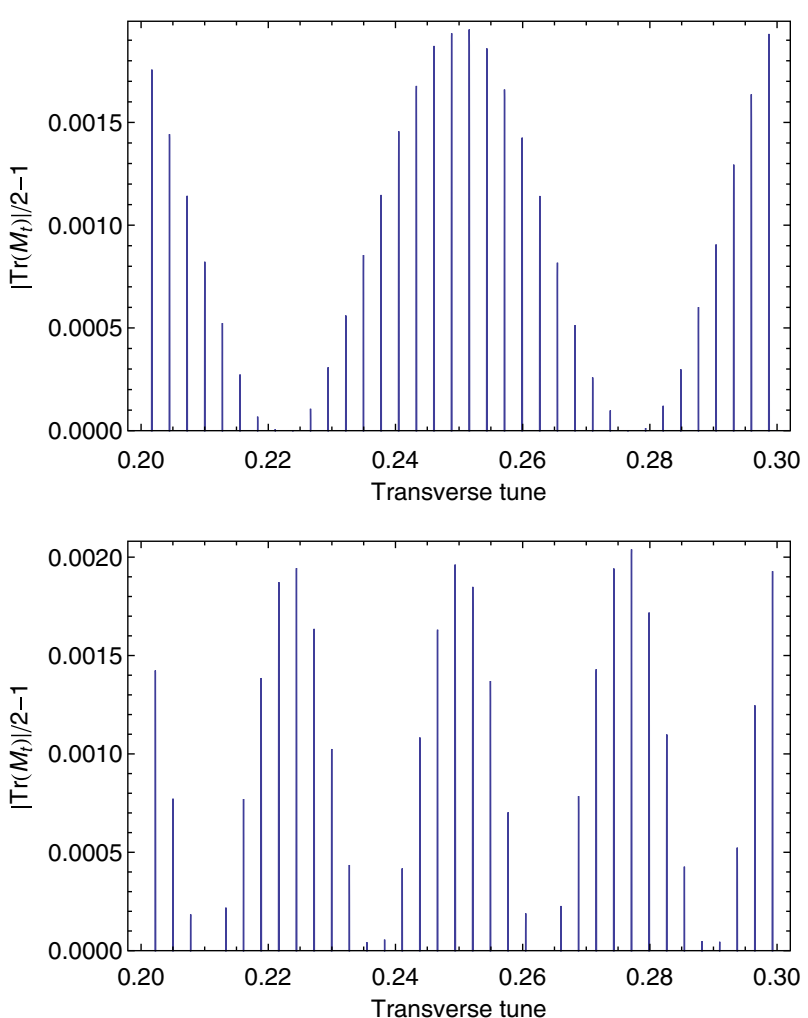

FIG. 7. $\left|\operatorname{Tr}\left(M_{t}\right)\right| / 2-1$ as function of betatron tune. The parameters used in calculation: beam-beam parameter is $0.01 ; N_{2}=180$. In the top two figures, $m=171$ and $n=9$; in the bottom two figures, $m=161$ and $n=19$. The right figures are the enlarged version of the left-hand ones. 


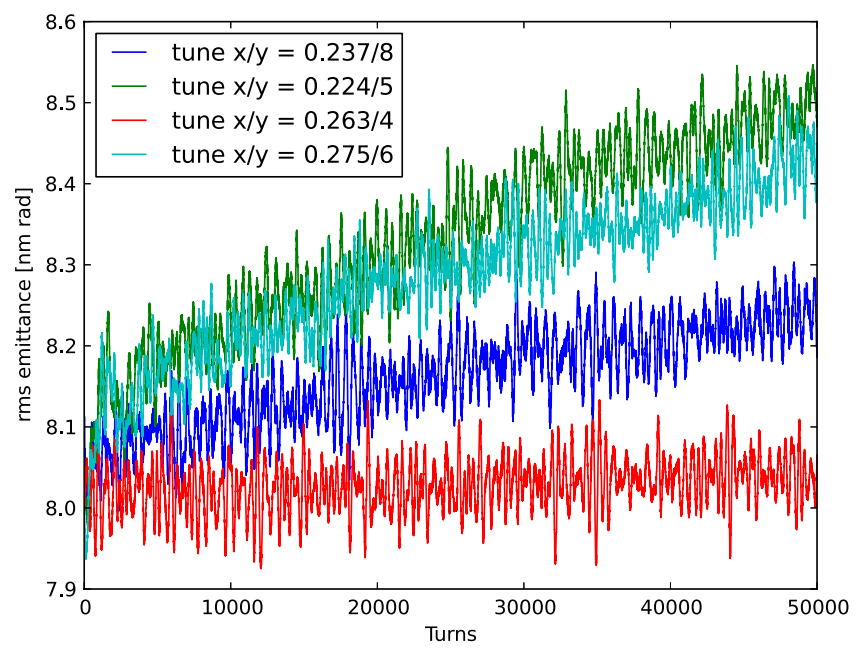

FIG. 8. Nonlinear 4D beam-beam simulation for different working points. The following parameters were used in calculation: beam-beam parameter is $0.01 ; N_{2}=180 ; m=161$, and $n=19$.

buckets ( $n$ in the above study). There will be only an $n-1$ stable region that can be considered. The effect implies another import limitation on the tune selection of both rings of the collider.

\section{SUMMARY}

We demonstrated the effective one-turn matrix with a permutation matrix and illustrated the formation of the multibunch resonance and the instability of both beam offsets and beam sizes in an asymmetric collision pattern when the number of bunches in two beams are different. We also evaluated the effect of the presence of the bunch gap.

This collision pattern induces new resonances that do not occur in a symmetric case. The separation of the resonance lines are approximately the reciprocal of the number of bunches in the ring, a feature that imposes a strong limitation on the stable beam-beam parameter even after proper working point selection, and therefore restricts the achievable luminosity. The countermeasure to the dipole offset resonance is to incorporate an ultrahigh gain bunch-bybunch transverse damper, which is a very challenging issue, even with state-of-the-art technology. The resonance and instability of the beam size of the colliding bunches will induce fast luminosity loss, and there are no obvious remedies. If there is a gap in the bunch train, the linear map for a single particle may become unstable, since the presence of $n$ continuous bunch gaps will create $n$ unstable regions of the tune space of the ring. The optimization of tune has different requirements for all three effects in the above discussion, which makes it almost impossible to find the proper value.

We conclude from our linear model and simplified nonlinear simulation that this asymmetric collision scheme (gear-changing scheme) would introduce a major loss of the luminosity in colliders. A more detailed strong-strong 6D beam-beam simulation (available tools include COMBI [5] or Beam-Beam 3D [6]) is needed to verify if the resonance can be suppressed by the Landau damping due to chromaticity [7] or nonlinear magnets. Such simulations are usually very time consuming and beyond the scope of this paper.

\section{APPENDIX: JUSTIFICATION OF THE ONE-TURN MATRIX}

In this appendix, we will prove the proposed one-turn matrix in Eq. (1), to the power of $N_{2}$, presenting the same spectrum as the natural superturn matrix, which is the one for $\operatorname{LCM}\left(N_{1}, N_{2}\right) / N_{1}$ turns for ring 1 or $\operatorname{LCM}\left(N_{1}, N_{2}\right) / N_{2}$ turns for ring 2. Assuming $N_{2}>N_{1}\left(\Delta N=N_{2}-N_{1} \ll\right.$ $N_{1}$ ), and they are relatively prime, the superturn matrix $O$ can be built as in Ref. [3]:

$$
\begin{aligned}
O= & K_{N_{1}, N_{2}} M_{s} K_{N_{1}-1, N_{2}-1} M_{s} \ldots K_{1, N_{2}-N_{1}+1} M_{s} \\
& \ldots \\
& K_{N_{1}, 2 N_{1}\left(\bmod N_{2}\right)} M_{s} \ldots K_{1, N_{1}+1} M_{s} \\
& K_{N_{1}, N_{1}} M_{s} \ldots K_{2,2} M_{s} K_{1,1} M_{s} .
\end{aligned}
$$

Here, the operation $\left(\bmod N_{2}\right)$ is different with a normal modulo operation, and always gives positive integers (i.e., we define $n N_{2} \bmod N_{2}=N_{2}$ for any integer $n$, instead of zero in normal modulo operation). The $K_{m, n}$ is the matrix for the collision between the bunch $m$ in ring 1 and bunch $n$ in ring 2 , which is a $2\left(N_{1}+N_{2}\right)$ rank identity matrix except for the following elements:

$$
\begin{aligned}
K_{m, n}[2 m, 2 m-1] & =1 / f_{b b}, \\
K_{m, n}[2 n, 2 n-1] & =1 / f_{b b}, \\
K_{m, n}[2 m, 2 n-1] & =-1 / f_{b b}, \\
K_{m, n}[2 n, 2 m-1] & =-1 / f_{b b},
\end{aligned}
$$

and $M_{s}$ is the transverse map of $N_{1}+N_{2}$ bunches with betatron phase advance $2 \pi \nu_{1} / N_{1}$ for ring 1 and $2 \pi v_{2} / N_{2}$ for ring 2 .

$M_{s}=\left(\begin{array}{cc}I\left(N_{1}\right) \otimes M_{\beta}\left(2 \pi \nu_{1} / N_{1}\right) & 0 \\ 0 & I\left(N_{2}\right) \otimes M_{\beta}\left(2 \pi \nu_{2} / N_{2}\right)\end{array}\right)$.

This construction of matrix $O$ is grouped to $N_{2}$ lines such that each line in the right-hand side contains matrices for $N_{1}$ collisions. The $(j+1)$ th line $\left(j=0 \ldots N_{2}-1\right)$ from the bottom can be expressed as

$$
\begin{aligned}
& K_{N_{1},(j+1) N_{1}\left(\bmod N_{2}\right)} M_{s} \ldots K_{1, j N_{1}+1\left(\bmod N_{2}\right)} M_{s} \\
& \quad=P_{T}^{-j} K_{N_{1}, N_{1}} M_{s} \ldots K_{1,1} M_{s} P_{T}^{j},
\end{aligned}
$$

where $P_{T}$ is the permutation matrix defined in Eq. (4). 
For the transverse map $M_{s}$, we can always rewrite it as $M_{s}=\prod_{i}^{N_{1}} m_{1, i} \prod_{i}^{N_{2}} m_{2, i} . m_{1, i}$ and $m_{2, i}$ are the transverse matrix for $N_{1}+N_{2}$ bunches with only zero phase advance except for the $i$ th bunch in ring 1 or ring 2 with its phase advance $\nu_{1} / N_{1}$ or $\nu_{2} / N_{2}$. It is obvious that $m_{1, i} m_{2, j}=$ $m_{2, j} m_{1, i}$ and $m_{1 / 2, i} K_{m, n}=K_{m, n} m_{1 / 2, i}$ if $i \neq m$ and $i \neq n$.

Therefore the $(j+1)$ th line can be rewrite as

$$
\begin{aligned}
& P^{-j} K_{N_{1}, N_{1}} M_{s} \ldots K_{1,1} M_{s} P^{j} \\
& =P^{-j} \prod_{i=N_{1}+1}^{N_{2}} m_{2, i}^{N_{1}} \prod_{i=1}^{N_{1}}\left(m_{1, i} m_{2, i}\right)^{N_{1}-i} \prod_{i=1}^{N_{1}} K_{i, i} \prod_{i=1}^{N_{1}}\left(m_{1, i} m_{2, i}\right)^{i} P^{j} .
\end{aligned}
$$

We use the property of the $P_{T}$ that $P_{T}^{N_{2}}=I$ and $P_{T} P_{T}^{T}=I$. The $O$ matrix then becomes

$$
\begin{aligned}
O= & {\left[P \prod_{i=N_{1}+1}^{N_{2}} m_{2, i}^{N_{1}} \prod_{i=1}^{N_{1}}\left(m_{1, i} m_{2, i}\right)^{N_{1}-i}\right.} \\
& \left.\prod_{i=1}^{N_{1}} K_{i, i} \prod_{i=1}^{N_{1}}\left(m_{1, i} m_{2, i}\right)^{i}\right]^{N_{2}} \equiv O_{N_{1}}^{N_{2}} .
\end{aligned}
$$

Now, we will prove that matrix multiplication $O_{N_{1}}$ in the above bracket has the same spectrum as the proposed oneturn matrix in Eq. (1). We will use the fact that $P m_{2, i}=m_{2, i-N_{1}\left(\bmod N_{2}\right)} P$. In addition, we know that the spectrum of the product of the square matrix is an invariant under cyclic permutation, $\sigma\left(A_{1} A_{2} \ldots A_{n}\right)=$ $\sigma\left(A_{2} A_{3} \ldots A_{n} A_{1}\right)$. Therefore, the following permuted matrix $O_{p 1}$ has the same spectrum as $O_{N_{1}}$ :

$$
\begin{aligned}
O_{p 1}= & P \prod_{i=1}^{N_{1}} K_{i, i} \prod_{i=1}^{N_{1}}\left(m_{1, i} m_{2, i}\right)^{i} \prod_{i=N_{1}+1}^{N_{2}} m_{2, i-N_{1}\left(\bmod N_{2}\right)}^{N_{1}} \\
& \times \prod_{i=1}^{N_{1}}\left(m_{1, i} m_{2, i-N_{1}\left(\bmod N_{2}\right)}\right)^{N_{1}-i} \\
= & P M_{k} \prod_{i=1}^{N_{1}}\left(m_{1, i}\right)^{N_{1}} \prod_{i=1}^{\Delta N}\left(m_{2, i}\right)^{N_{1}+i} \\
& \times \prod_{i=\Delta N+1}^{N_{1}} m_{2, i}^{N_{2}} \prod_{i=N_{1}+1}^{N_{2}} m_{2, i}^{N_{2}-j} .
\end{aligned}
$$

[1] The Relativistic Heavy Ion Collider (RHIC) at Brookhaven National Laboratory is an example.

[2] The Electron Light Ion Collider (ELIC) proposed by Jefferson Laboratory is an example.

[3] K. Hirata and E. Keil, Nucl. Instrum. Methods Phys. Res., Sect. A 292, 156 (1990).

[4] K. Yokoya et al., Part. Accel. 27, 181 (1990).

[5] T. Pieloni, Report No. CERN-THESIS-2010-056, 2008.

[6] J. Qiang, M. A. Furman, and R. D. Ryne, J. Comput. Phys. 198, 278 (2004)

[7] Y. Alexahin, Nucl. Instrum. Methods Phys. Res., Sect. A 480, 253 (2002). 